\title{
Efficient Multi-Ported Memories for FPGAs
}

\author{
Charles Eric LaForest and J. Gregory Steffan \\ Department of Electrical and Computer Engineering \\ University of Toronto \\ \{laforest,steffan\}@eecg.toronto.edu
}

\begin{abstract}
Multi-ported memories are challenging to implement with FPGAs since the provided block RAMs typically have only two ports. We present a thorough exploration of the design space of FPGA-based soft multi-ported memories by evaluating conventional solutions to this problem, and introduce a new design that efficiently combines block RAMs into multi-ported memories with arbitrary numbers of read and write ports and true random access to any memory location, while achieving significantly higher operating frequencies than conventional approaches. For example we build a 256location, 32-bit, 12-ported (4-write, 8-read) memory that operates at $281 \mathrm{MHz}$ on Altera Stratix III FPGAs while consuming an area equivalent to 3679 ALMs: a $43 \%$ speed improvement and $84 \%$ area reduction over a pure ALM implementation, and a $61 \%$ speed improvement over a pure "multipumped" implementation, although the pure multipumped implementation is $7.2 \mathrm{x}$ smaller.
\end{abstract}

\section{Categories and Subject Descriptors}

B.3.2 [Memory Structures]: Design Style-Shared Memory

\section{General Terms}

Design Performance

\section{Keywords}

FPGA, memory, multi-port, parallel

\section{INTRODUCTION}

As FPGAs continue to increase in transistor density, designers are using them to build larger and more complex systems-on-chip that require frequent sharing, communication, queueing, and synchronization among distributed functional units and compute nodes. For ASIC implementations these mechanisms would often be implemented with multi-ported memories - memories that allow multiple reads and writes to occur simultaneously_-since they can avoid serialization and contention. For example, processors normally require a multi-ported register file: more register file ports allows the processor to exploit a greater amount of instruction-level parallelism (ILP) where multiple instructions are being executed at the

Permission to make digital or hard copies of all or part of this work for personal or classroom use is granted without fee provided that copies are not made or distributed for profit or commercial advantage and that copies bear this notice and the full citation on the first page. To copy otherwise, to republish, to post on servers or to redistribute to lists, requires prior specific permission and/or a fee.

FPGA'10, February 21-23, 2010, Monterey, California, USA

Copyright 2010 ACM 978-1-60558-911-4/10/02 ...\$10.00.

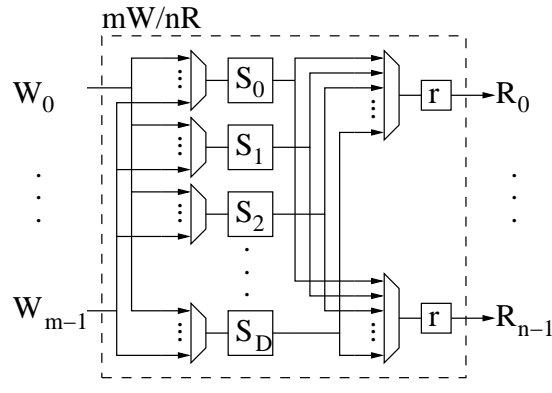

Figure 1: A multi-ported memory implemented with FPGA logic blocks, having $D$ single-word storage locations $(S), m$ write $(W)$ ports, and $n$ read $(R)$ ports (encoded as $m W / n R$ ), and $n$ temporary registers $r$. Only read and write data lines are shown (i.e., not address lines).

same time. However, FPGA-based soft processors have so far exploited little ILP, limited mainly to simple instruction pipelines. This is partly due to the fact that multi-ported memories are particularly inefficient to implement using the resources typically provided by FPGAs.

\subsection{Conventional Approaches}

It is possible to implement a multi-ported memory using only the basic logic elements of an FPGA, as illustrated in Figure 1, which shows a $D$-location memory with $m$ write ports and $n$ read ports. As shown, we require $D m$-to-one decoders to steer writes to the appropriate memory locations, and $n D$-to-one multiplexers to allow each read to access any memory location. Note also that the read outputs are registered $(r)$ to implement a synchronous memory where the output is held stable between clock edges. The problem is that this circuit scales very poorly, with area increasing rapidly with memory depth and the decoding/multiplexing severely limiting the maximum operating frequency.

It is normally more efficient to implement memories on FPGAs using the provided block RAMs, each of which can be quite large (e.g., 9Kbits) while supporting high operating frequencies (e.g., $580 \mathrm{MHz}$ ). However, FPGA block RAMs currently provide only two ports for reading and/or writing. Note that Altera's Mercury line of Programmable Logic Devices (PLDs) [2] previously provided quad-port RAMs to support gigabit telecom applicationshowever, this feature has not been supported in any other Altera device, likely due to the high hardware cost.

System designers have hence used one or a combination of three conventional techniques for increasing the effective number of ports of FPGA block RAMs, as shown in Figure 2. The first is replication, which can increase the number of read ports by maintaining a replica of the memory for each additional read port. However, 


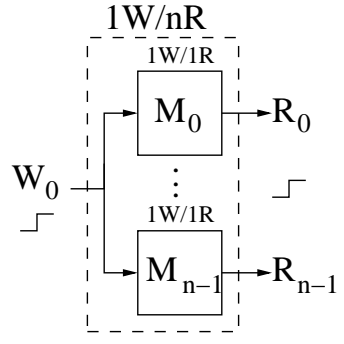

(a) Replication

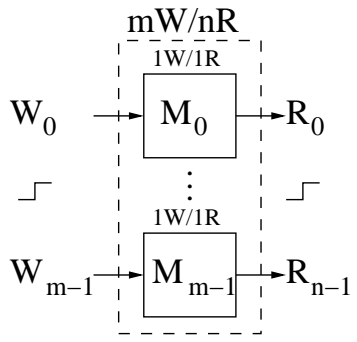

(b) Banking

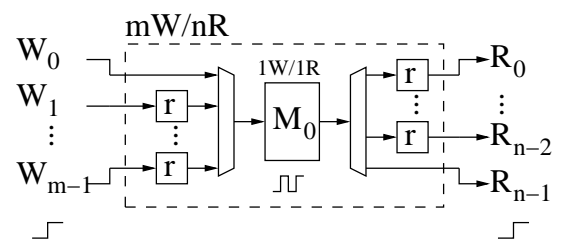

(c) Multipumping

Figure 2: Three conventional techniques for providing more ports given a $1 \mathrm{~W} / 1 \mathrm{R}$ memory (read and write address values are not depicted, only data values): (a) Replication maintains an extra copy of the memory to support each additional read port, but is limited to supporting only one write port; (b) Banking divides data across multiple memories, but each read or write port can only access one specific memory; (c) Multipumping multiplies the number of read/write ports of a memory by adding internal data and address multiplexers and temporary registers $(r)$, and internally clocking the memory at a multiple of the external clock (which quickly degrades the maximum external operating frequency).

this technique alone cannot support more than one write port, since the one external write port must be routed to each block RAM to keep it up-to-date. The second is banking, which divides memory locations among multiple block RAMs (banks), allowing each additional bank to support an additional read and write port. However, with this approach each read or write port can only access its corresponding memory division-hence a pure banked design does not truly support sharing across ports. The third we call "multipumping", where any memory design is clocked at a multiple of the external clock, providing the illusion of a multiple of the number of ports. For example, a $1 \mathrm{~W} / 1 \mathrm{R}$ memory can be internally clocked at $2 \mathrm{X}$ the external frequency to give the illusion of being a $2 \mathrm{~W} / 2 \mathrm{R}$ memory. A multipumped design must also include multiplexers and registers to temporarily hold the addresses and data of pending reads and writes, and must carefully define the semantics of the ordering of reads and writes. While reasonably straight-forward, the drawback of a multipumped design is that each increase in the number of ports dramatically reduces the maximum external operating frequency of the memory.

\subsection{A More Efficient Approach}

In this paper we propose a new design for true multi-ported memories that capitalizes on FPGA block RAMs while providing (i) substantially better area scaling than a pure logic-based approach, and (ii) higher frequencies than the multipumping approach. The key to our approach is a form of indirection through a structure called the Live Value Table (LVT), which is itself a small multiported memory implemented in reconfigurable logic similar to Figure 1. Essentially, the LVT allows a banked design to behave like a true multi-ported design by directing reads to appropri- ate banks based on which bank holds the most recent or "live" write value. The intuition for why an LVT-based design is more efficient even though the LVT is purely implemented in logic elements is because the LVT is much narrower than the actual memory banks since it only holds bank numbers rather than full data values - thus the lines that are decoded/multiplexed are also much narrower and hence more efficiently placed and routed. An LVTbased design also leverages block RAMS, which implement memory more efficiently, and has an operating frequency closer to that of the block RAMs themselves. Additionally, LVT-based design and multipumping are complementary, and we will show that with multipumping we can reduce the area of an LVT-based design by halving its maximum operating frequency. With these techniques we can support soft solutions for multi-ported memories without expensive hardware block RAMs with more than two ports.

\subsection{Related Work}

There are several prior attempts to implement multi-ported memories in the context of FPGAs, mainly for the purpose of soft processor register files. Most soft uniprocessors exploit replication to provide the $1 \mathrm{~W} / 2 \mathrm{R}$ register file required to support a three-operand ISA [6-8, 13, 17]. Jones et al. [9] implement a VLIW soft processor where additional register file ports support a zero-overhead interface to custom hardware functions. However, their multi-ported register file is implemented entirely in the FPGA's reconfigurable logic and limits the operating frequency of their soft processor. Saghir et al. $[14,15]$ implement a multi-ported register file for a VLIW soft processor by exploiting both replication and banking; however, this requires that the compiler schedule register accesses such that there are not two simultaneous reads or writes to the same bank. Nonetheless, this approach is sufficient to support multithreading $[10,12,13]$ since each thread need only read/write its own division of the register file. Manjikian exploits an aggressive form of multipumping by performing reads and writes on consecutive rising and falling clock edges within a processor cycle [11]. His approach avoids Write-After-Read (WAR) violations by performing all writes before reads. Unfortunately this design requires that the entire system use multiple-phase clocking.

\subsection{Contributions}

This paper makes the following contributions: (i) we present the first thorough exploration of the design space of FPGA-based soft multi-ported memories; (ii) we evaluate conventional methods of building such memories and confirm that they do not scale well; (iii) we introduce the Live Value Table (LVT), an efficient mechanism for implementing multi-ported memories with an arbitrary number of read and write ports; (iv) we demonstrate that LVT-based designs are smaller and faster than pure reconfigurable logic implementations, as well as faster and more scalable than pure multipumping implementations; (v) we evaluate the impact of multipumping on LVT-based designs, and demonstrate that they are complementary.

\section{EXPERIMENTAL FRAMEWORK}

Memory Designs We consider only memories of 32-bit element width as this is the common case in many computing systems. We consider a range of multi-ported memory designs that have at least one write port and two read ports $(1 \mathrm{~W} / 2 \mathrm{R})$ such that all ports are usable simultaneously within a single external cycle. We do not consider one-write-one-read (1W/1R) memories as they are trivial to implement with a single FPGA block RAM. We also do not consider memories that may stall (eg., take multiple cycles to return 
read values should they conflict with concurrent writes), although such designs would be compelling future work. Additionally, we assume that multiple writes to the same address are prevented by the system using the multi-ported memory, and that the result of doing so is undefined. Each design is wrapped in a test harness such that all paths begin and end at registers, allowing us to ensure proper timing analysis and to test each design for correctness. The Verilog sources are generic and do not contain any Altera-specific modules or annotations.

CAD FLow We use Altera's Quartus 9.0 to target the Altera Stratix III EP 3SL340F1760C2, a large and fast device that allows us to compare with published results for the Nios II soft processor [5]. We do not bias the synthesis process to favour area or speed, nor perform any circuit transformations such as retiming. We configured the place and route process to make a standard effort at fitting with only two constraints: (i) to avoid I/O pin registers to prevent artificially long paths that would affect the clock frequency, and (ii) to set the target clock frequency to $1 \mathrm{Ghz}$ to optimize circuit layout for speed ${ }^{1}$. We report maximum operating frequency by averaging the result of place/routes across ten different random seeds.

Measuring Area We report area as the total equivalent area, which estimates the actual silicon area of a design point: we calculate the sum of all the Adaptive Logic Modules (ALMs) plus the area of the Block RAMs counted as their equivalent area in ALMs ${ }^{2}$. Each ALM can contain unrelated logic and registers, avoiding an inflated logic utilization measure due to underused ALMs.

\section{STRATIX III ARCHITECTURE}

The following describes the basic components provided by the Altera Stratix III architecture. Although our work targets Altera's Stratix III FPGAs, the following concepts generally translate to the devices of other FPGA vendors. For example, other than the different capacities, the block RAMs in Xilinx's Virtex-6 FPGAs would function identically.

Adaptive Logic Module (ALM) Memory FPGAs can implement memory using their generic reconfigurable logic composed of Adaptive Logic Modules (ALMs). The Stratix III ALMs each contain two registers, some adder logic, and Look-Up Tables (LUTs). ALM memory has virtually no constraints on capacity, configuration, and number of ports, but pays a large area and speed penalty (Figure 1). The CAD tools may also require a prohibitive amount of time (over an hour) to place and route such a memory.

Block RAM (BRAM) Memory FPGAs implement block RAMs directly on their silicon substrate. Block RAMs have two ports that can each function either as a read or a write port. These memories use less area and run at a higher frequency than ones created from the FPGA's reconfigurable logic, but do so at the expense of having a fixed storage capacity and number of ports. The Stratix III FPGA devices mostly contain M9K block RAMs ${ }^{3}$, which hold nine kilobits of information in various widths and depths. At a width of 32 bits, an M9K holds 256 elements.

\footnotetext{
${ }^{1}$ This approach was recommended by an experienced user of Quartus as more practical than iterated guessing.

${ }^{2}$ Altera graciously provided the confidential area equivalence of BRAMs for Stratix II. We extrapolated the unavailable Stratix III area numbers from the Stratix II data and other confidential data.

${ }^{3}$ They also contain larger M144K block RAMs which each hold 144 kilobits (as $4 \mathrm{k} \times 32$ for example), but exist in much fewer numbers than M9Ks and target bulk RAM instead.
}

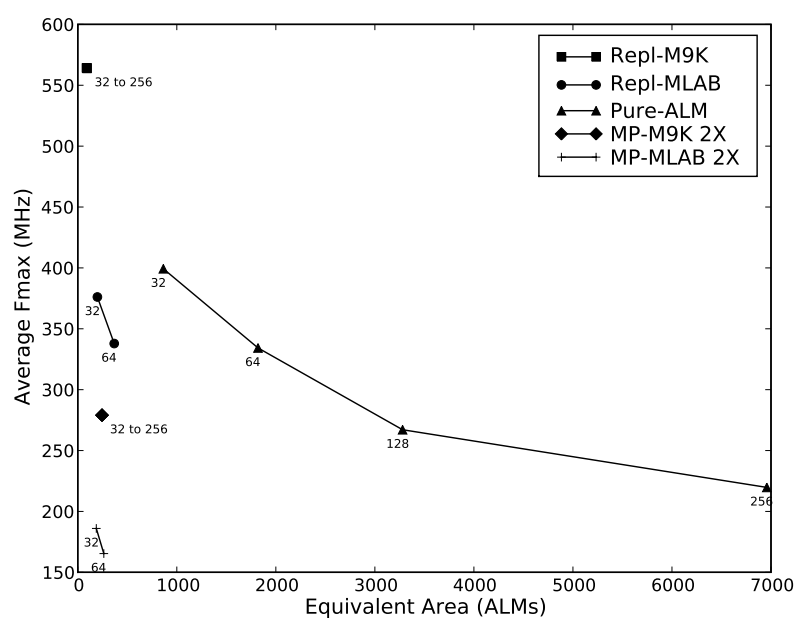

Figure 3: Comparison of the speed and area of various ALM, M9K, and MLAB implementations of 32-bit 1W/2R memories of varying depth (as indicated by the number at each data point). The prefix denotes the implementation technique: "Pure" for pure logic, "Repl" for replication, and "MP" for pure multipumping. The smallest possible M9K designs have a capacity of 256 elements, hence the two M9K designs are all overlapping. Placement/routing fail for MLAB designs of depth greater than 64.

Memory Logic Array Block (MLAB) Memory The Stratix III FPGA architecture clusters its ALMs into Logic Array Blocks (LABs), each containing ten ALMs. Some of the LABs can function either as a group of ALMs or as a single small block of memory, or Memory LAB (MLAB). MLABs provide a halfway point between ALM and BRAM implementations: they are small, numerous, and widely distributed like ALMs, but implement memory in a denser, constrained manner like BRAMs. A single MLAB holds up to 20 words of 16 bits. Unlike other memories, which perform all operations on the rising edge of the clock, MLABs read on the rising edge and write on the falling edge. MLABs best implement small shift registers and FIFO buffers and not arbitrarily deep memories.

\section{CONVENTIONAL MULTI-PORTING}

A simple two-ported memory, with one read and one write port (1W/1R) defines the basic conceptual and physical unit of storage from which we build multi-ported memories. We assume that each port may access any one location per cycle, and if a read and write to the same location occur in the same cycle, the read port obtains the current contents of the location and the write port overwrites the contents at the end of the cycle ("Write-After-Read" (WAR) operation).

The simplest multi-ported memory that we consider is a $1 \mathrm{~W} / 2 \mathrm{R}$ memory. This memory is interesting because it is not naturally supported by FPGA structures but is commonly used, for example for soft processor register files. Figure 3 plots the area and operating frequency of $1 \mathrm{~W} / 2 \mathrm{R}$ memories of varying depth (where the depth is indicated by the number next to each point), and of varying implementation. We use these results to discuss the following conventional techniques for building multi-ported memories on FPGAs:

Pure ALMs A straightforward method for constructing a multiported memory on an FPGA is to do so directly in ALMs-i.e., a design like that shown in Figure 1. We evaluate such designs in Figure 3, shown as the Pure-ALM series of points. From the figure we see that even a 32 -entry $1 \mathrm{~W} / 2 \mathrm{R}$ memory requires 864 ALMs 
for this design. As we increase depth, area increases rapidly and operating frequency drops significantly. This trend motivates the need to use block RAMs for more efficient multi-ported memories.

Replication Replication (Figure 2(a)) is an easy way to increase the number of read ports of a simple memory (i.e., to $1 \mathrm{~W} / \mathrm{nR}$ ): simply provide as many copies of the memory as you require read ports, and route the write port to all copies to keep them up-to-date. We evaluate replication in Figure 3 for both M9Ks (Repl-M9K) and MLABs (Repl-MLAB). All of the Repl-M9K designs fit into two M9K BRAMs, such that those points are all co-located in the figure. Replication requires no additional control logic, hence these designs are very efficient. For $1 \mathrm{~W} / 2 \mathrm{R}$ memories with a depth greater than 256 elements, another pair of M9Ks would be added at every depth increment of 256 elements-resulting in a relatively slow increase in area as memory depth increases. We also consider replicated designs composed of MLABs (Repl-MLAB). Unfortunately, Quartus could not place and route any MLAB-based memory with more than 64 elements. Since each MLAB stores the equivalent of 160 ALMs, the Repl-MLAB implementation requires much less interconnect than the Pure-ALM implementation but considerably more than the Repl-M9K implementation. For example, the 32entry Repl-MLAB 1W/2R memory requires only 198 equivalent ALMs, but still suffers a lower operating speed of $376 \mathrm{MHz}$. The replicated M9K designs (Repl-M9K) are evidently far superior to the alternatives, with an area of 90 equivalent ALMs and maximum operating frequency of $564 \mathrm{MHz}$. However, the drawback to this approach is that there is no way to provide additional write ports with replication alone-we must pursue other techniques to provide more write ports.

Banking Banking (Figure 2(b)) is similar to replication, except that the memory copies are not kept coherent; each additional memory now supports an additional read and write port, providing an easy way to increase ports arbitrarily ( $\mathrm{mW} / \mathrm{mR})$. The conventional way to use banking is to divide memory locations evenly among the banks, such that each read and write port are tied to a certain memory division. However, a memory with only banking is not truly multi-ported, since only one read from a certain division is possible in a given cycle. For this reason we do not evaluate banked-only memories, although a close estimate of the Fmax/area of a mW/mR banked memory is the corresponding $1 \mathrm{~W} / \mathrm{mR}$ replicated design.

Multipumping Multipumping (Figure 2(c)) internally uses an integer multiple of the external system clock to multiplex a multiported memory with fewer ports, giving the external appearance of a larger number of ports $(\mathrm{mW} / \mathrm{nR})$. This requires the addition of multiplexers and registers to hold temporary states, as well as the generation of an internal clock, and careful management of the timing of read-write operations. We further describe the details of implementing a multipumped design in the next section.

\subsection{Multipumping Implementations}

Since multipumped memories multiplex ports over time, the order of read/write operations must be carefully managed: violating the precedence of reads and writes would break the external appearance of them occurring at the same time. In particular, writes must be performed at the end to avoid Write-After-Read (WAR) violations where an earlier internal write updates a value before it has been read by a subsequent internal read.

For non-multipumped designs, each block RAM port supports either a read or a write, hence we use the block RAMs in "simple dual-port" mode where a port is statically defined to be for reading or writing. Since multipumped designs time-multiplex the block RAM ports we can potentially exploit "true dual-port" mode, where a block RAM port can be dynamically configured for reading or writing. For the simplest multipumped design consisting of a single block RAM, true dual-port mode can allow us to configure both ports for reads and perform pairs of reads until all are done, then configure both ports as writes and perform pairs of writes until all are done.

A larger but more aggressive multipumped design can also exploit banking to reduce the number of cycles required to perform reads: each bank can perform two unique reads, and all banks can operate in parallel; when reads are completed, one pair of writes can be performed across all banks each cycle until all writes are performed. In other words, the block RAMs are read like a banked memory and are written like a replicated memory. Similar techniques have been published by Xilinx [16] and Actel [1] but only for certain forms of quad-port memories, whereas our implementation supports arbitrary numbers of read and write ports.

True dual-port mode is not free: for Stratix III FPGAs [3] an M9K block RAM in simple dual-port mode has 256 locations of 32 bits, while in true dual-port mode it has 512 locations of 16 bits since the RAM output drivers are split to support two reads. Therefore true dual-port mode requires two M9K block RAMs to create a 32-bit-wide memory. Despite this doubling, the number of block RAMs required remains practical: even an 8W/16R purely multipumped memory would need only one block RAM pair to support each read port, for a total of 32 .

The following summarizes the design of a pure multi-ported memory using true dual-port mode for the block RAMs. Given an arbitrary $\mathrm{mW} / \mathrm{nR}$ memory, the number of cycles required to perform all the $m$ writes and $n$ reads follows $\lceil m / 2+n / 2 x\rceil$, where $x$ counts the number of block RAMs. The $m / 2$ term stems from each write being replicated to all the block RAMs to avoid data fragmentation, making the whole memory appear to have only two write ports. The $n / 2 x$ term comes from each block RAM being able to service any two reads at once since the writes replicate their data to all block RAMs. The ceiling function handles cases where there are either more internal ports than there are external read or write ports, or the number of internal ports does not evenly divide the number of external ports. A fractional number of cycles in a term implies that, for one of the cycles, some ports remain free and some writes might be done simultaneously with the last reads. The typical case is when the number of block RAMs equals the number of read ports, allowing all reads to be performed in one cycle while leaving half the ports available for one of the writes, which may save one cycle in certain port configurations. Larger numbers of block RAMs will not further reduce the number of cycles.

As a simple example, in Figure 3 we implement 1W/2R memories by double-pumping M9Ks (MP-M9K 2X) and MLABs (MPMLAB 2X) ${ }^{4}$. While $2 \mathrm{X}$ multipumping does halve the number of M9Ks or MLABs used, the overhead of the required control circuitry negates any area savings for memories with so few ports. The maximum external operating frequencies of the double-pumped designs are also a little under half those of the replicated designs (186 MHz for MP-MLAB 2X, and $279 \mathrm{MHz}$ for MP-M9K 2X). As we will demonstrate later, multipumping can be an important technique to reduce area when building memories with larger numbers of ports.

\footnotetext{
${ }^{4}$ Again, due to Quartus' difficulty with MLABs, the multipumping implementation uses simple dual-port MLABs only. For $1 \mathrm{~W} / 2 \mathrm{R}$ only, this does not affect the area or external operation.
} 


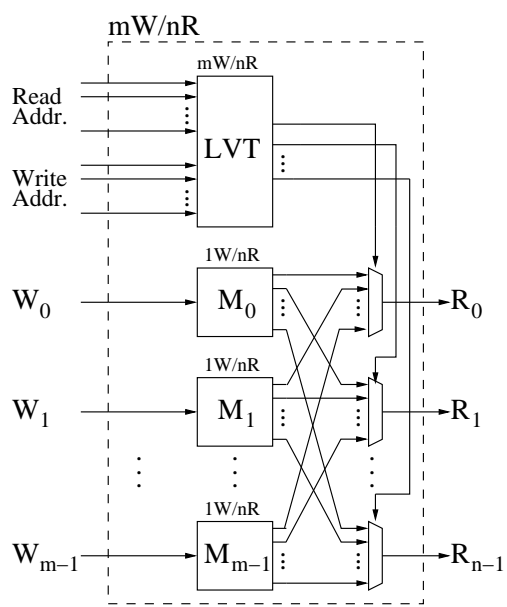

Figure 4: A generalized $\mathrm{mW} / \mathrm{nR}$ memory implemented using a Live Value Table $(L V T)$. Each write updates its own replicated memory bank $(M)$ and updates its entry at the same address in the LVT. For each read, the LVT selects the memory bank that holds the most recently written value for the requested memory address.

\subsection{Summary}

A $1 \mathrm{~W} / 2 \mathrm{R}$ memory can easily be extended to have more read ports by increasing the amount of replication, but this technique cannot be used to add more write ports. While banking easily allows multiple write ports, such designs must map reads and writes to divisions of the memory, and do not allow true sharing. A multiported memory implemented purely in ALMs scales poorly. Multipumping by itself causes a large drop in operating frequency. In the next section, we introduce a method for transparently managing and keeping coherent banked memories to effectively allow multiple read and write ports.

\section{LVT-BASED MULTIPORTED MEMORIES}

We propose a new approach to implementing multi-ported memories on FPGAs that can exploit the strengths of all three conventional techniques for adding ports. Our approach comprises banks of replicated block RAMs where a mechanism of indirection steers each read to the bank holding the most-recent write value. Multipumping is orthogonal to our approach, and can be applied to reduce the area of a memory in cases where a slower operating frequency can be tolerated, as we demonstrate later in Section 7. We name our indirection mechanism the Live Value Table (LVT), since it tracks which bank contains the "live" or mostrecently updated value for each memory location. A brief outline of this approach is described by Altera [4], but provides no details of operation, no comparisons, and limits itself to only four ports.

\subsection{The Basic Idea}

Figure 4 illustrates an LVT-based multi-ported memory. The memory is composed of $m$ banks ( $M_{0}$ to $M_{m-1}$ ), each of which contains a $1 W / n R$ memory (constructed via replication of block RAMs) such that $n$ is equal to the desired number of read ports ( $R_{0}$ to $\left.R_{n-1}\right)$. Each write port writes to its own bank, and each read port can read from any of all the banks via its multiplexer. The banked memory allows for arbitrary concurrent writes, while the replication within each bank supports arbitrary concurrent reads. The LVT is a $\mathrm{mW} / \mathrm{nR}$ multi-ported memory implemented using ALMs.

At a high level, the design operates as follows. During a write to a given address, the write port updates that location in its block

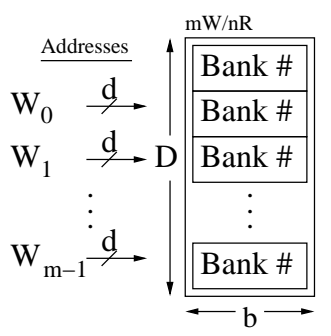

(a) Write Operation

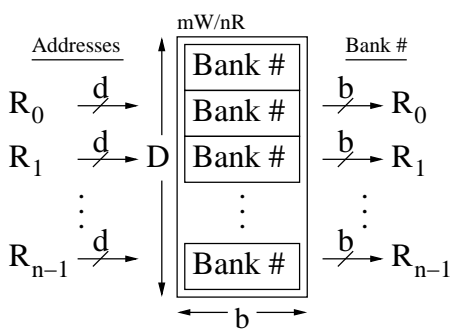

(b) Read Operation
Figure 5: A Live Value Table (LVT) for a multi-ported memory of depth $D$ with $m$ write ports $(W)$ and $n$ read ports $(R)$. Each LVT location corresponds to a memory location, and tracks the bank number of the memory bank that holds the most recent write value. Every write updates the corresponding location with the destination bank number, and every read is directed to the appropriate bank by the bank number stored in the corresponding LVT location. The width $(b)$ of the bank numbers is $\log _{2}(m)$. The width $(d)$ of the addresses is $\log _{2}(D)$.

RAM bank with the new value, and the LVT simultaneously updates its corresponding location with the bank number ( 0 to $m-1)$. During a read, the read port sends the address to every bank and to the LVT. All the banks return their value for that location and the LVT returns the number of the write port which last updated that location, driving the multiplexer of the read port to select the output of the proper block RAM bank.

\subsection{Implementing the LVT}

Figure 5 illustrates the overall structure and operation of a LVT for a multi-ported memory of depth $D$ with $m$ write ports $(W)$ and $n$ read ports $(R)$. Each LVT location corresponds to a memory location, and tracks the bank number of the memory bank that holds the most recent write value for that memory location. Despite being implemented entirely in ALMs, the area of a LVT remains tractable due to its narrow width $b=\log _{2}(m)$. For example, compared to the 864 ALMs of the 32-element $1 \mathrm{~W} / 2 \mathrm{R}$ Pure-ALM memory in Figure 3, a LVT of the same depth with $2 \mathrm{R} / 2 \mathrm{~W}$ ports uses only $75 \mathrm{ALMs}^{5}$. Even with $8 \mathrm{~W} / 16 \mathrm{R}$ ports, the corresponding LVT consumes only 649 ALMs.

During writes, the LVT uses the memory write addresses to update the corresponding locations with the numbers of the ports performing the writes. These numbers identify the block RAM banks that hold the written values. During reads, the LVT uses the read addresses to fetch the bank numbers that in turn steer the outputs of those banks to the read ports. All addresses are of width $d=\log _{2}(D)$.

\subsection{LVT Operation}

As an example of the operation of a Live Value Table, Figure 6 depicts two writes and two reads to a multi-ported memory similar to the one depicted in Figure 4. The memory contains one memory bank for each write port $\left(W_{0}\right.$ and $\left.W_{1}\right)$. Each memory bank is a replicated block RAM memory with enough ports for each read port $\left(R_{0}\right.$ and $\left.R_{1}\right)$. The LVT at the top is implemented using ALMs only, has the same depth as each memory bank, but stores the much narrower bank numbers. The write ports place their bank number in the LVT at the same address at which they write their data to the banks. The LVT controls the output multiplexer of each read port. The memory begins empty or otherwise uninitialized.

\footnotetext{
${ }^{5} \mathrm{~A} 2 \mathrm{~W} / 2 \mathrm{R}$ LVT is the smallest meaningful case here, as a memory with a single write port does not need an LVT.
} 


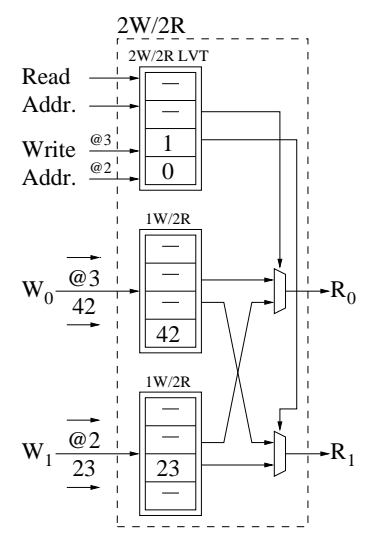

(a) Write Operation

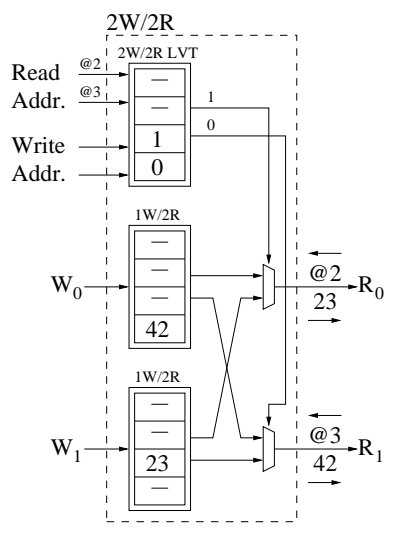

(b) Read Operation

Figure 6: Example operation of a 2W/2R LVT-based multiported memory: during write operation, $W_{0}$ writes 42 to address 3 and $W_{1}$ writes 23 to address 2 , and the LVT records for each address the bank that was last written; during read operation, $R_{0}$ reads address 2 and $R_{1}$ reads address 3 , and the LVT selects the appropriate bank for each read address.

Figure 6(a) shows the state of the memory banks and the LVT after port $W_{0}$ writes the value 42 to address 3 and port $W_{1}$ writes 23 to address 2 . The values are stored into the separate memory banks of ports $W_{0}$ and $W_{1}$, while the LVT stores their bank numbers at the same addresses.

An access from any read port will simultaneously send the address to the LVT and to each memory bank. The bank number returned by the LVT directs the output multiplexer to select the output of the block RAM memory bank containing the most current value for the second memory element. In Figure 6(b), port $R_{1}$ reads from address 3 and thus gets 42 from bank 0 , while port $R_{0}$ reads from address 2 and gets 23 from bank 1 .

\subsection{Block RAM Requirements}

Having memory banks which can hold the entire memory contents for each write port and having each of these banks internally replicated once for each read port means that the total number of block RAMs within all the banks equals the product of the number of write ports and read ports, times the number of block RAMs necessary to hold the entire memory contents in a single bank. For example, the rather large case of a 32-bit 8W/16R multi-ported memory requires 128 block RAMs for depths of up to 256 elements. Even the smallest Stratix III FPGA (EP 3SL 50) contains 108 M9K block RAMs, while mid-range devices contain 275 to 355. Also, the relatively large depth of the M9K block RAMs allows correspondingly large multi-ported memories to be implemented. Larger memories would likely require the use of deeper block RAMs such as the Stratix M144K. In Section 7, we will demonstrate how multipumping can reduce the number of required block RAMs.

\subsection{Recursive LVT Implementation}

An LVT implements a multi-ported memory using ALMs and thus grows proportionately with depth-however, since each location stores only the few bits required to encode a memory bank number, the memory size remains practical. It would seem desirable to repeat this area-saving and implement the LVT itself using block RAMs, managed by a still smaller, inner LVT. However, we cannot avoid implementing a LVT using ALMs since FPGAs do not provide any suitable multi-ported block RAMs with enough write ports and the narrow width of an LVT. Ideally, a number of $\mathrm{mW} / 1 \mathrm{r}$ block RAMs could be used as a replicated memory to create

a mW/nR LVT without the use of ALM-based storage, but no such block RAMs exist on FPGAs. Additionally, any inner LVT used to coordinate block RAMs implementing a larger outer LVT would necessarily be implemented using ALMs and would have the same depth and control the same number of banks and ports as the outer LVT it sought to replace. This inner LVT would thus have the same area as the outer LVT, and hence is not worth it.

\section{LVT PERFORMANCE}

While an LVT does solve the problem of adding write ports to a memory, it also introduces additional delay due to the bank number look-up and the read port multiplexers, and increases the area due to internal replication of each memory bank. In this section and the next, we demonstrate that the LVT-based approach provides (i) substantially better area scaling than a pure logic-based approach, and (ii) higher frequencies than multipumping approaches.

\subsection{Speed vs. Area}

Figure 7(a) and Figure 8(a) plot the average maximum operating frequency (Fmax) versus area for $2 \mathrm{~W} / 4 \mathrm{R}$ and $4 \mathrm{~W} / 8 \mathrm{R}$ memories of increasing depth (denoted by the number next to the data point). It is apparent that the pure ALM implementation (Pure-ALM) is inefficient: for the 4W/8R memory, 32 elements requires 3213 ALMs and 256 elements requires 23767 ALMs. The larger of these pure ALM designs are likely impractially large for most applications.

Looking at the MLAB-based LVT implementations (LVT-MLAB) for 2W/4R, the designs are smaller but achieve a slower Fmax than the corresponding pure ALM designs. For the 4W/8R designs, the MLAB-based LVT implementations are both larger and slower than the corresponding pure ALM designs. Furthermore, the MLABbased designs cannot support memories deeper than 64 elements since Quartus cannot place and route them. Overall the MLABbased designs are uncompelling, except for providing an area-Fmax trade-off relative to the pure ALM designs for 2W/4R memories.

From the figures it is evident that the M9K-based implementations are superior. The area of the $2 \mathrm{~W} / 4 \mathrm{R}$ and 4W/8R LVT-M9K implementations increases much more slowly with depth than the pure ALM implementation. Furthermore, as an indication of their usability, these designs achieve a clock frequency close-to or better than the $290 \mathrm{MHz}$ clock frequency of a NiosII/f soft processor on the same Stratix III device [5]. For example, the 4W/8R version has an operating frequency ranging from $361 \mathrm{MHz}$ at 32 elements, down to $281 \mathrm{MHz}$ for 256 elements, with enough ports to support four such soft processors.

\subsection{Area Breakdown}

Figure 7(b) and Figure 8(b) display the total equivalent area of various implementations of the same $2 \mathrm{~W} / 4 \mathrm{R}$ and $4 \mathrm{~W} / 8 \mathrm{R}$ memories, broken down into their components. The Pure-ALM implementation is a single multi-ported memory without any specified subcomponents: the synthesis process implements all of the multiplexers, decoders, and storage implicitly. These increase in proportion with the depth of the memory and rapidly become impractically large.

The LVT-MLAB implementation, despite using denser memory, suffers from higher interconnect area overhead. The area of the LVT-MLAB memory banks increases quickly with the memory depth since each MLAB can only store 20 words of 16 bits. Also, Quartus could not place and route MLAB-based memories deeper than 64 elements. The absence of output multiplexers for the 64element $2 \mathrm{~W} / 4 \mathrm{R}$ memory is due to a fortuitous synthesis optimization by Quartus: each register in an ALM has two load lines, which may eliminate the multiplexer when there are only two sources.

The LVT-M9K block RAM Memory Banks have the lowest area 


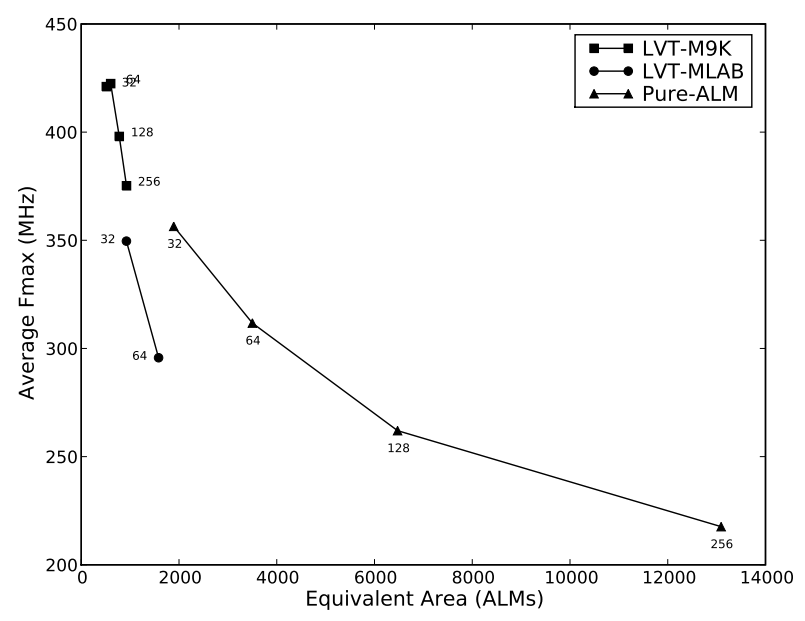

(a) Fmax vs Area

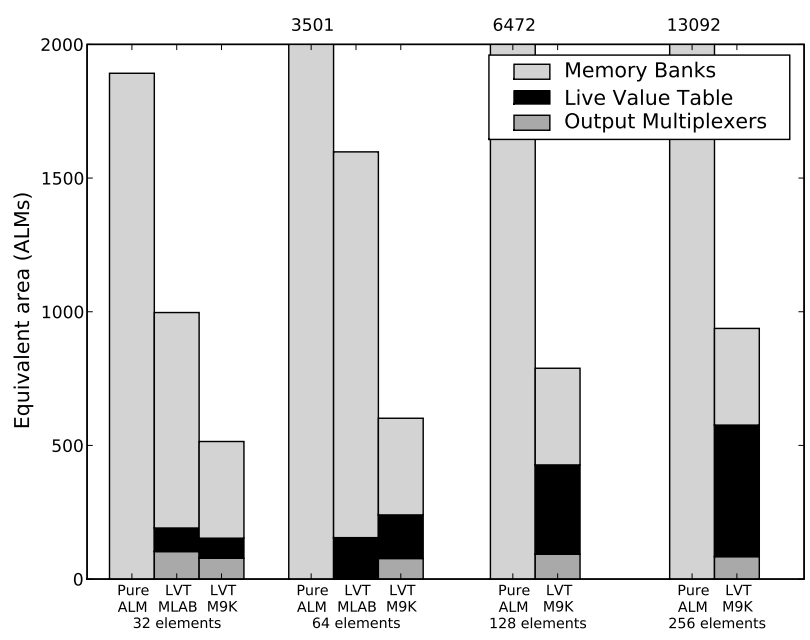

(b) Area Breakdown

Figure 7: Speed and area for Pure-ALM, LVT-MLAB, and LVT-M9K implementations of a 2W/4R memory with an increasing number of memory elements.

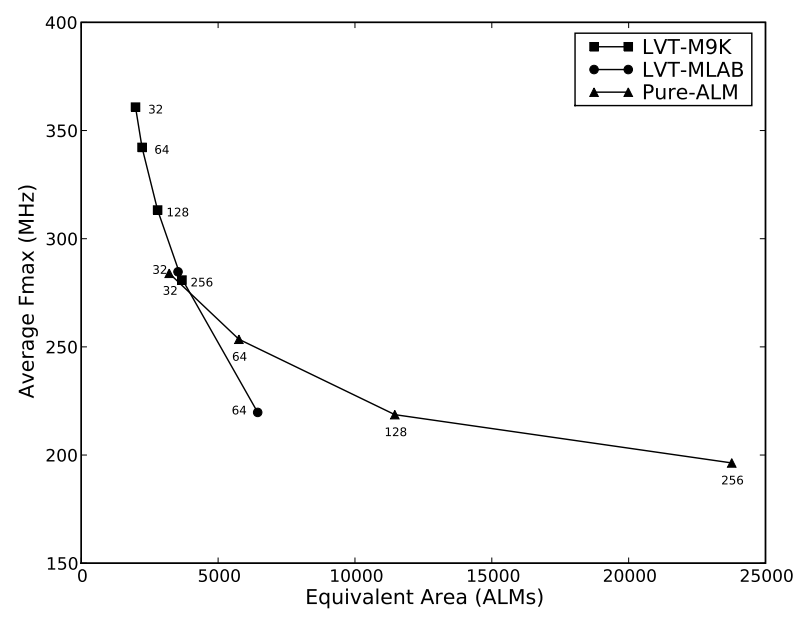

(a) Fmax vs Area

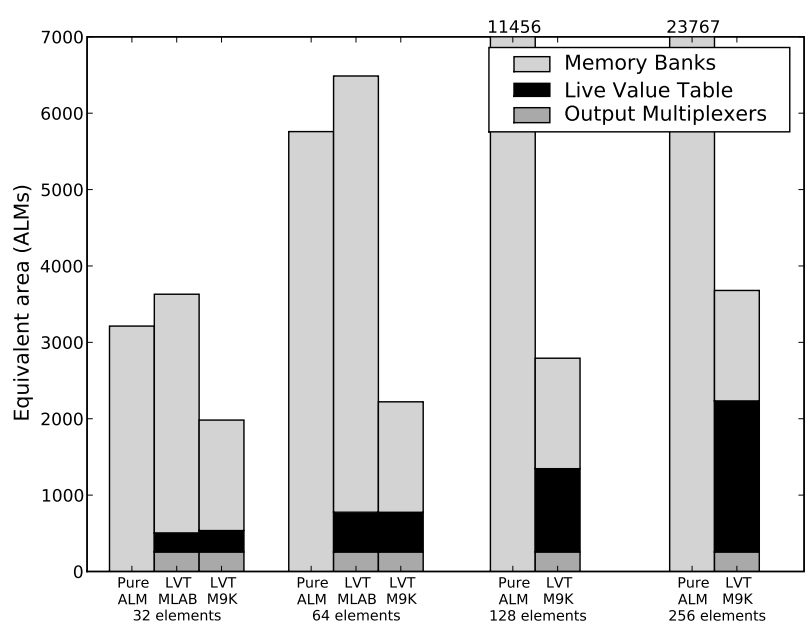

(b) Area Breakdown

Figure 8: Speed and area for Pure-ALM, LVT-MLAB, and LVT-M9K implementations of a 4W/8R memory with an increasing number of memory elements.

due to their higher density and lower interconnect requirements. Most of the multiplexing and decoding overhead in the Pure-ALM and LVT-MLAB implementations becomes implicit in the circuitry of the M9K block RAMs. The area of the LVT-M9K 4W/8R Memory Banks remains constant at 1446 equivalent ALMs since all of the memory depths fit into the same number of block RAMs. Even with the non-trivial overhead of the LVT, the LVT-M9K implementations consume much less total area than the alternatives.

The LVTs of the LVT-MLAB and LVT-M9K implementations have the exact same internal structure and the same depth as the corresponding Pure-ALM memory implementation and thus also scale proportionately with the depth of the memory. However, the LVTs only store the one or two bits required to identify a memory bank, reducing their growth to tractable levels. As an example, the area of the LVT of the LVT-M9K 4W/8R memory ranges from 280 ALMs up to 1977 ALMs: approximately one-tenth the area of the corresponding Pure-ALM memory. The area of the $4 \mathrm{~W} / 8 \mathrm{R}$ output multiplexers, when present, remains constant at 256 ALMs since the number of banks in the LVT-MLAB and LVT-M9K memories also remains constant. For the $2 \mathrm{~W} / 4 \mathrm{R}$ memory, the multiplexer area fluctuates between 77 and 93 ALMs, likely due to optimizations made possible when an ALM has inputs from only two banks.

\section{MULTIPUMPING PERFORMANCE}

In the previous section we observed that M9K implementations of LVT-based multi-ported memories are faster and smaller than the alternatives - for some applications the achievable Fmax is potentially overkill. In such cases we could apply multipumping (introduced earlier in Section 4) to trade Fmax for reduced area as the application allows. In this section we describe and measure multipumping applied to LVT-based designs, and also compare with pure multipumping-based multi-ported memory designs.

\subsection{Speed vs. Area}

Multipumping can bring about a useful reduction in area if the speed of the original memory is significantly higher than required by the surrounding system. Figure 9(a) and Figure 10(a) compare the maximum external operating frequency (Fmax) and the total area of M9K-based LVT 2W/4R and 4W/8R memories with $2 \mathrm{X}$ and $4 \mathrm{X}$ multipumping, along with the equivalent pure multipump- 


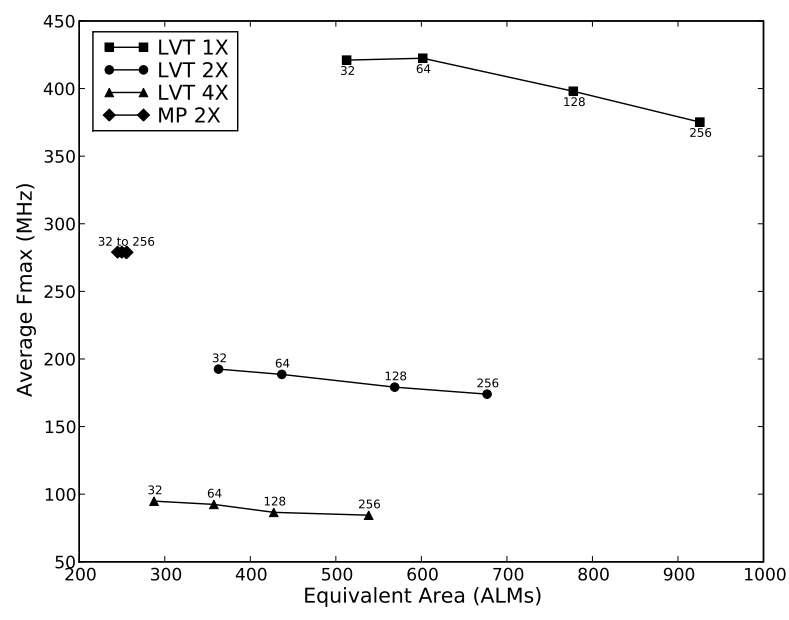

(a) Fmax vs Area

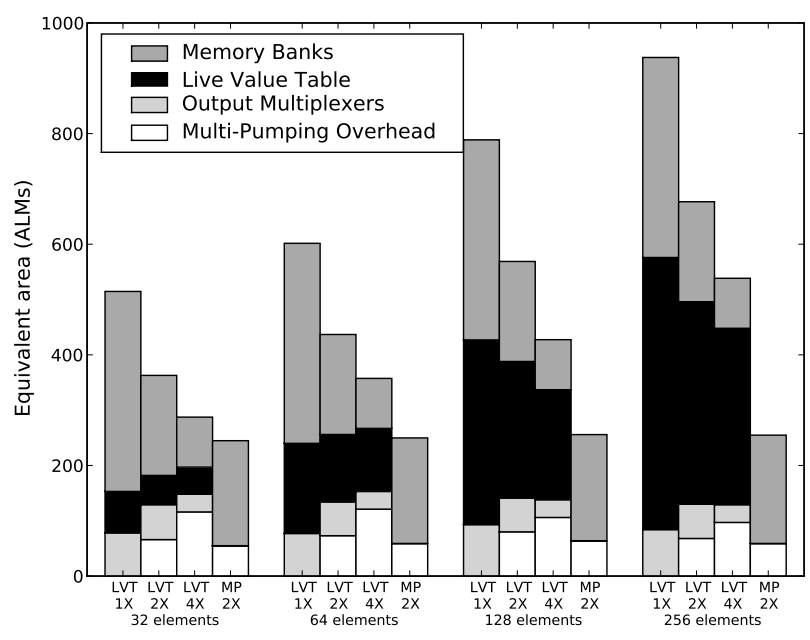

(b) Area Breakdown

Figure 9: Speed and area for M9K-based 2W/4R multipumped memories: an LVT memory with multipumping factors of 1X (a 2W/4R memory with no multipumping), $2 X$ (a $2 W / 2 R$ memory with two internal cycles), and $4 X$ (a $2 W / 1 R$ memory with four internal cycles), and a pure multipumping memory (MP 2X).

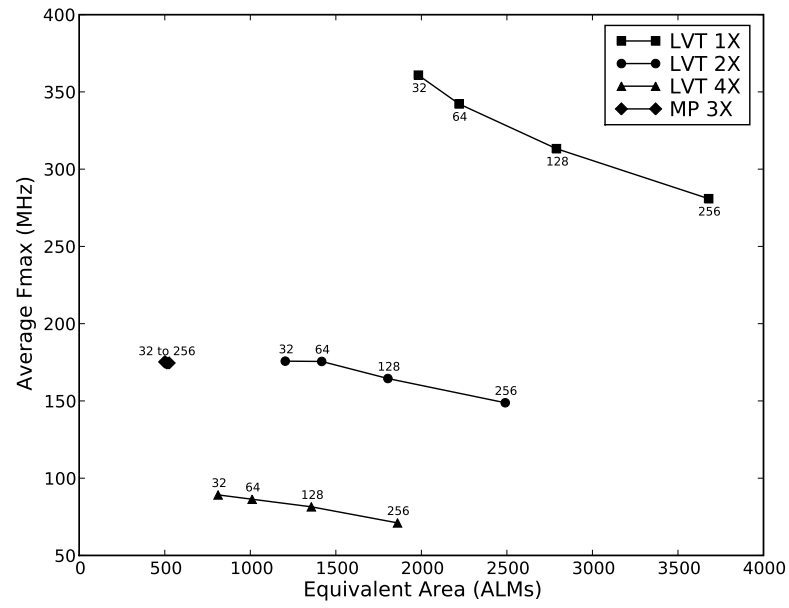

(a) Fmax vs Area

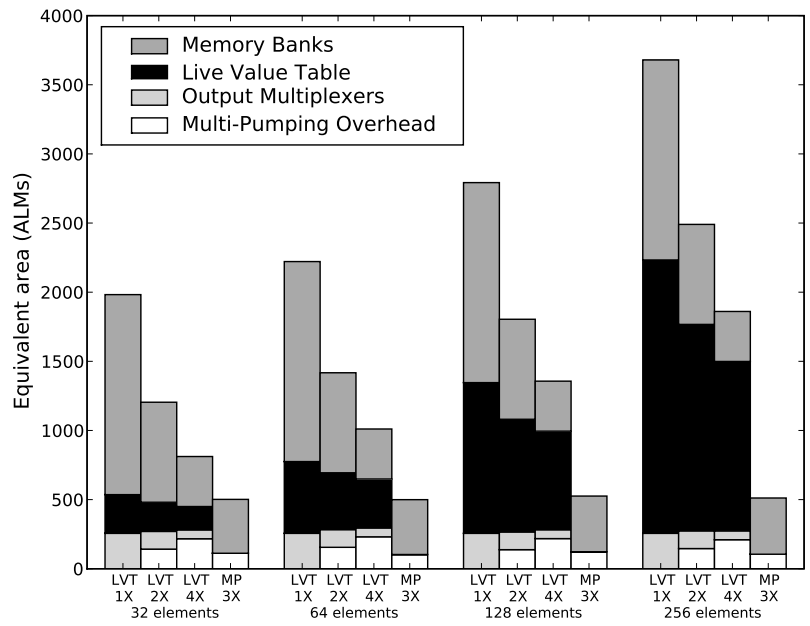

(b) Area Breakdown

Figure 10: Speed and area for M9K-based 4W/8R multipumped memories: an LVT memory with multipumping factors of 1X (a 4W/8R memory with no multipumping), 2X (a 4W/4R memory with two internal cycles), and 4X (a 4W/2R memory with four internal cycles), and a pure multipumping memory (MP 3X).

ing (MP) implementations. For all cases, the internal operating frequency remains approximately equal to the Fmax of the original baseline memory prior to multipumping, which ranges for the LVT 4W/8R memory from $361 \mathrm{MHz}$ to $281 \mathrm{MHz}$ as the depth increases, and $523 \mathrm{MHz}$ for all depths of the MP $3 \mathrm{X}$ 4W/8R memory.

Despite the high internal operating frequencies, dividing them by a multipumping factor does bring about a harsh external speed penalty. For example, the 4W/8R LVT 2X multipumped implementations in Figure 10(a) operate externally at frequencies ranging from $176 \mathrm{MHz}$ to $149 \mathrm{MHz}$, which may still be practical speeds. The MP 3X implementations also hold at $174 \mathrm{MHz}$. For either implementation, it is evident that only small multipumping factors can be used before the drop in Fmax becomes too great to be practical. Although we have tested multipumping factors of up to eight, we expect that most designs will use a factor of two or three.

Furthermore, although the pure multipumping (MP) implementations seem to have better performance and a greatly reduced area, a multipumping factor of two is only possible for $1 \mathrm{~W} / 2 \mathrm{R}$ (Figure 3 ) and 2W/4R memories (Figure 9(a)). Pure multipumping memories with more ports will always require a multipumping factor of at least three or four, which quickly drops the Fmax. By comparison, a multipumping factor of two is always feasible for any LVT memory with an even number of read ports. The slower drop in speed of an LVT memory as the number of ports increases (Figure 9(a) vs. Figure 10(a)) is a consequence of its internal parallelism, instead of the mostly serial operation of a pure multipumping memory.

\subsection{Area Breakdown}

The primary benefit of multipumping is reducing the area of the memory banks at the expense of clock frequency. Although the area of the memory banks reduces proportionally to the amount of multipumping, the LVT does not scale down as much and limits the overall area reduction.

As discussed in Section 5.4, the number of block RAMs in a multi-ported LVT memory is equal to the product of the number of read and write ports. Since multipumping divides the number of 
internal read ports, the number of block RAMs per bank is reduced by the same factor ${ }^{6}$. The number of read ports on the Live Value Table reduces to match, as does the number of output multiplexers. Figure 9(b) and Figure 10(b) show how multipumping affects the area of each of these components for the same LVT $2 \mathrm{~W} / 4 \mathrm{R}$ and $4 \mathrm{~W} / 8 \mathrm{R}$ memories when using factors of two (2X) and four (4X), compared to a factor of one (1X) as the baseline non-multipumped case, which is identical to the LVT-M9K bars of Figure 7(b) and Figure 8(b). The figures also show the area breakdown of the equivalent pure multipumping (MP) memories.

For LVT memories, the multipumping factor exactly divides the area of the memory banks by itself since now only one-half or one-quarter the number of internal read ports exists, which also reduces the area of the output multiplexers by the same ratio. For the $4 \mathrm{~W} / 8 \mathrm{R}$ memory, the area of the Live Value Table shrinks by only $24 \%$ for $2 \mathrm{X}$ and $36 \%$ for $4 \mathrm{X}$ on average since its number of write ports remains unchanged". The "Multipumping Overhead" fraction contains the additional overhead of multipumping such as the Multipumping Controller, internal multiplexers, and temporary registers. Regardless of the depth of the memory, multipumping introduces a small, nearly constant overhead: 145 ALMs for 4W/8R LVT $2 X$ multipumping, and 219 ALMs for 4W/8R LVT 4X on average. Summed together, these individual changes to the $4 \mathrm{~W} / 8 \mathrm{R}$ LVT memories reduce the total area by an average of $36 \%$ for $2 \mathrm{X}$ multipumping, and $54 \%$ for $4 \mathrm{X}$. The unchanged number of write ports in the LVT primarily limits how much we can reduce the area.

The pure multipumping memories (MP) use much less area since they do not require a Live Value Table or Output Multiplexers, nor use as many block RAMs since their banks are not replicated. For example, the 4W/8R MP 3X memory in Figure 10(b) uses only eight M9K block RAMs inside a total equivalent area of 511 ALMs, of which 105 are multipumping overhead. Unfortunately, pure multipumping memories tend to have higher minimum multipumping factors and thus slower Fmax than LVT memories as the number of ports increases. In Section 8.1, we will explore the idea of using pure multipumping memories with a small number of ports to potentially improve the efficiency of LVT-based memories.

\section{MORE AGGRESSIVE DESIGNS}

In this section we describe potential design avenues that are more aggressive than those we have presented: a way to build an even more efficient LVT-based design, and relaxing read/write ordering to ease constraints on the design of the multi-ported memory.

\subsection{LVT-Based Memory Based on Pure Mul- tipumped Banks}

If even moderately multi-ported block RAMs became available on FPGAs, some very significant area improvements to LVT-based multi-ported memories would follow. For example, doubling the number of read and write ports on a block RAM would mean needing only half as many memory banks to support the write ports of an LVT-based memory, with each bank containing only half as many replicated memories to service the read ports, resulting in needing only a quarter of the number of block RAMs to construct a given LVT-based multi-ported memory. Furthermore, halving the number of banks reduces the width of the LVT by one bit, which is significant since a typical LVT is only three bits wide or less.

\footnotetext{
${ }^{6}$ This assumes the multipumping factor can evenly divide the number of read ports. For example, a 4W/8R LVT memory supports factors of two, four, or eight only.

${ }^{7}$ This fact suggests that the narrower but more numerous write port multiplexers and decoders have the largest impact on the area of pure ALM memories.
}

Although most FPGAs do not provide block RAMs with more than two ports, some of the smaller pure multipumping memories might provide usable substitutes. This speculation is supported by the interesting performance of the 'MP $2 X^{\prime}$ ' $2 \mathrm{~W} / 4 \mathrm{R}$ pure multipumping design from Figure 9: 255 equivalent ALMs at $279 \mathrm{MHz}$, using four M9K block RAMs. If we used this memory to construct the banks of the 'LVT $1 \mathrm{X}$ ' 4W/8R LVT-based memory in Figure 10, two banks would be required instead of four, with each bank internally replicated once to support the read ports for a total of four 2W/4R memories. This sums to only 16 M9K block RAMs instead of 32, and even with the additional area overhead of multipumping $^{8}$ the area of the memory banks would decrease by $29 \%$, while the area of the LVT would be halved. It is easy to see from Figure 10(b) that these changes would significantly reduce the area of the 256-element 4W/8R 'LVT 1X' implementation. The impact on speed is harder to predict due to the large changes in the structure of the memory banks, but it is conceivable that the operating frequency would remain near that of the underlying $2 \mathrm{~W} / 4 \mathrm{R}$ pure multipumping memory.

\subsection{Relaxed Read/Write Ordering}

The primary obstacle to getting the most area benefit from multipumping is the relatively small area reduction of the LVT since the number of write ports cannot be divided. The writes must all occur together after the reads to prevent WAR violations. If we relax the $\mathrm{read} / \mathrm{write}$ ordering and allow writes to occur before all of the reads have completed, then time-multiplexing the internal write ports becomes possible. The multipumping factor can now divide both the number of internal memory banks and the number of write ports on the Live Value Table, further improving the area reduction.

For example, with a multipumping factor of two and the read/write ordering preserved, our $4 \mathrm{~W} / 8 \mathrm{R}$ multi-ported memory example internally becomes a 4W/4R memory. Halving the number of read ports only halves the size of the memory banks and reduces the size of the LVT to a lesser degree. By comparison, if we allow relaxed read/write ordering, then the multipumping factor can also divide the number of write ports ${ }^{9}$, which will in turn divide the number of memory banks in addition to their size and further reduce the area of the LVT. In effect, except for the small overhead of the multipumping control circuitry, the entire 4W/8R memory would internally reduce to a $2 \mathrm{~W} / 4 \mathrm{R}$ instance which uses about $75 \%$ less hardware. This quadratic area reduction is immediately visible when comparing the LVT entries in Figure 9(b) and Figure 10(b), as well as the LVT-M9K entries in Figure 7(b) and Figure 8(b).

Relaxing the read/write ordering requires the designer to schedule the reads and writes to the multi-ported memory to avoid WAR violations which would corrupt data. For example, given our 4W/8R example multi-ported memory with a multipumping factor of two and relaxed read/write ordering, the reads and writes will internally execute as two consecutive $2 \mathrm{~W} / 4 \mathrm{R}$ sets, each using one half of the external ports. If the designer wants to simultaneously read and write to the same location within a system cycle, both operations must be grouped in the same read/write set by performing them on the appropriate external ports. If the designer cannot rearrange them, then the write operations must explicitly occur after the conflicting reads, either by placing them in the following read/write set, or in the next system cycle. Fortunately, this problem is identical to dependence analysis for optimizing software loops.

\footnotetext{
${ }^{8}$ This is pessimistic. For example, all of the multipumped memories could share a single multipumping controller.

${ }^{9}$ This assumes that the multipumping factor can evenly divide the number of write ports.
} 


\section{CONCLUSIONS}

FPGA systems provide efficient block RAMs, but with only two ports. Conventional approaches to building memories on FPGAs with a larger number of ports are either very area inefficient, slow, or both. We introduced a smaller and faster implementation for multi-ported memories based on the Live Value Table (LVT) - a small, narrow, multi-ported memory implemented in logic elements that coordinates read and write accesses such that a banked memory design to behave like a true multi-ported design. The resulting multi-ported memories provide true Write-After-Read (WAR) random access to any value, from an arbitrary number of ports, without the need to schedule reads and writes.

For example, using a LVT controlling 32 M9K block RAMs, we were able to implement a 256-element 12-ported (4W/8R) multiported memory which operates at $281 \mathrm{MHz}$ on Altera Stratix III FPGAs while consuming an area equivalent to 3679 ALMs: a $43 \%$ speed improvement and $84 \%$ area reduction over the equivalent pure ALM implementation, and a $61 \%$ speed improvement over a pure multipumping implementation, despite being $7.2 x$ larger. The higher speeds of our LVT-based designs presented the possibility of exchanging speed for area by applying multipumping. On average, $2 \mathrm{X}$ multipumping reduced the total area by $36 \%$, while $4 \mathrm{X}$ did so by $54 \%$. Our designs also allowed for lower and more practical multipumping factors than pure multipumping implementations as the number of ports increased.

We also proposed two potential avenues for further increasing the efficiency of LVT-based designs: (i) relaxing the ordering of reads and writes which avoided WAR violations would increase the area reduction from multipumping to about $75 \%$ at $2 \mathrm{X}$, minus the overhead of multipumping, at no additional cost in speed; (ii) implementing the memory banks of a $4 \mathrm{~W} / 8 \mathrm{R}$ LVT-based memory using $2 \mathrm{~W} / 4 \mathrm{R}$ pure multipumping memories could reduce the area of the memory banks by $29 \%$ and halve the area of the LVT while conceivably keeping the operating frequency in a useful range.

In summary, our exploration of the design space led us to three main conclusions: (i) LVT-based multi-ported memories are superior to logic-element-based designs in both area and speed; (ii) LVT-based implementations are faster than pure multipumping implementations although with an area cost; (iii) pure multipumping implementations can be sufficient if the number of required ports or external operating frequency are modest.

\section{REFERENCES}

[1] Implementing Multi-Port Memories in ProASIC ${ }^{\text {PLUS }}$ Devices. http: / / www . actel.com/documents / APA_MultiPort_AN.pdf, July 2003. Application Note AC176, Accessed Sept. 2009.

[2] Mercury Programmable Logic Device Family Data Sheet. http://www.altera.com/literature/ds / dsmercury . pdf, Jan 2003. Version 2.2, Accessed Sept. 2009.

[3] Stratix III Device Handbook Volume 1, Chapter 4: TriMatrix Embedded Memory Blocks in Stratix III Devices. http://www.altera.com/literature/hb/ stx3/stx3_siii51004.pdf, May 2008. Version 1.8, Accessed Sept. 2009.

[4] Advanced Synthesis Cookbook: A Design Guide for Stratix II, Stratix III, and Stratix IV Devices.

http://www.altera.com/literature/manual/ stx_cookbook.pdf, July 2009. Version 5.0, Accessed Nov. 2009.
[5] Nios II Performance Benchmarks. http://www.altera.com/literature/ds/ds_ nios2_perf.pdf, June 2009. Version 4.0, Accessed Sept. 2009.

[6] Nios II Processor Reference Handbook. http://www.altera.com/literature/hb/ nios2/n2cpu_nii5v1.pdf, March 2009. Version 9.0, Accessed Sept. 2009.

[7] CARLI, R. Flexible MIPS Soft Processor Architecture. Tech. rep., Massachusetts Institute of Technology, Computer Science and Artificial Intelligence Laboratory, June 2008.

[8] Fort, B., Capalija, D., VRanesic, Z., and Brown, S. A Multithreaded Soft Processor for SoPC Area Reduction. In IEEE Symposium on Field-Programmable Custom Computing Machines (April 2006), pp. 131-142.

[9] Jones, A. K., Hoare, R., Kusic, D., FazeKas, J., AND Foster, J. An FPGA-based VLIW processor with custom hardware execution. In International Symposium on Field-Programmable Gate Arrays (2005).

[10] LabrecQue, M., AND StefFAn, J. Improving Pipelined Soft Processors with Multithreading. In International Conference on Field Programmable Logic and Applications (Aug. 2007), pp. 210-215.

[11] Manjikian, N. Design Issues for Prototype Implementation of a Pipelined Superscalar Processor in Programmable Logic. In PACRIM 2003: IEEE Pacific Rim Conference on Communications, Computers and Signal Processing (Aug. 2003), vol. 1, pp. 155-158 vol.1.

[12] Moussali, R., Ghanem, N., And Saghir, M. Microarchitectural Enhancements for Configurable Multi-Threaded Soft Processors. In International Conference on Field Programmable Logic and Applications (Aug. 2007), pp. 782-785.

[13] Moussali, R., Ghanem, N., And Saghir, M. A. R. Supporting multithreading in configurable soft processor cores. In CASES '07: Proceedings of the 2007 international conference on Compilers, Architecture, and Synthesis for Embedded Systems (New York, NY, USA, 2007), ACM, pp. 155-159.

[14] SAghiR, M., And NAOUs, R. A Configurable Multi-ported Register File Architecture for Soft Processor Cores. In ARC 2007: Proceedings of the 2007 International Workshop on Applied Reconfigurable Computing (March 2007), Springer-Verlag, pp. 14-25.

[15] SaghiR, M. A. R., El-Majzoub, M., ANd AKL, P. Datapath and ISA Customization for Soft VLIW Processors. In ReConFig 2006: IEEE International Conference on Reconfigurable Computing and FPGAs (Sept. 2006), pp. $1-10$.

[16] SaWyer, N., AND Defossez, M. Quad-Port Memories in Virtex Devices. http:

//www.xilinx.com/support/documentation/ application_notes/xapp228.pdf, September 2002. XAPP228 (v1.0), Accessed Sept. 2009.

[17] Yiannacouras, P., Steffan, J. G., And Rose, J. Application-specific customization of soft processor microarchitecture. In FPGA '06: Proceedings of the 2006 ACM/SIGDA 14th international symposium on Field Programmable Gate Arrays (New York, NY, USA, 2006), ACM, pp. 201-210. 